\title{
Charging mechanism of $\mathrm{AlGaN} / \mathrm{GaN}$ open-gate $\mathrm{pH}$ sensor and electrolyte interface
}

\author{
R. Anvari ${ }^{1,2, \dagger}$, M. Myers ${ }^{2}$, G. A. Umana-Membreno ${ }^{1}$, M. Baker ${ }^{2}$, D. Spagnoli ${ }^{2}$, G. Parish ${ }^{1}$, B. Nener ${ }^{1}$ \\ ${ }^{1}$ School of Electrical, Electronics and Computer Engineering, University of Western Australia \\ ${ }^{2}$ School of Chemistry and Biochemistry,University of Western Australia \\ †Electronic mail: roozbeh.anvari@research.uwa.edu.au
}

\begin{abstract}
The charging mechanism of the interface between an $\mathrm{AlGaN} / \mathrm{GaN}$ based open-gate ion-sensitive field-effect transistor and electrolyte is studied theoretically. Density functional theory calculations are performed to obtain the energy minimum structure of the surface oxide and electrolyte interface. Thermodynamics based relations are employed to obtain the double layer parameters. An analytical model is applied to study the carrier density modulation of the $\mathrm{AlGaN} / \mathrm{GaN}$ heterostructure by the influence of the surface charge.
\end{abstract}

keywords- AIGaN, GaN, Electrical Double Layer, Gallium Oxide, pH sensor, Density Functional Theory

\section{INTRODUCTION}

AlGaN/GaN open gate sensors have attracted significant interest for measuring the $\mathrm{pH}$ of electrolytes. The adsorption of the $\mathrm{H}^{+}$ions on the device native oxide surface results in surface charge accumulation that modulates the carrier density in the channel. However, the impact of the surface adsorption processes on the sensitivity of the sensor is not well understood. This study provides more insight into the charging mechanism of the sensor-surface and provides accurate estimation of the $\mathrm{Ga}_{2} \mathrm{O}_{3}$ protonation properties such as the number of surface sites and dissociation rates. Fig. 1, depicts the charging mechanism of the sensor-surface through protonation and deprotonation of the surface adsorption sites.

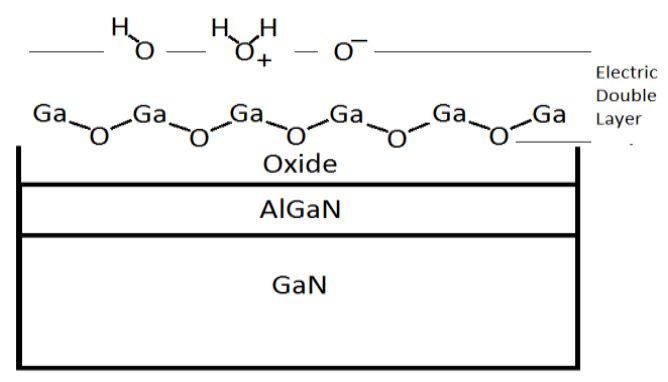

Fig. 1. Surface charging mechanism. Electrical double layer is used to model the interfacial charge distribution.

\section{MethodOLOGY}

The atomic coordinates, energetics of the relaxed structure and the surface site density are calculated through $a b$ initio simulations. The relaxed atomic coordinates are then used to calculate the surface reaction rates and interfacial capacitance. These parameters are used to derive the surface potential and charge density. Finally an analytical model is used to obtain the sensitivity of the sensor.

\section{A. ab Initio Calculations}

Electronic structure calculations of neutral $\alpha$-gallium oxide slabs in the [0001] direction were performed using Quantum Espresso [5]. Norm-conserving pseudo-potentials and Local Density Approximation (LDA) exchange and correlation functions as proposed by Pedrew-Zunger [5] were employed. A kinetic energy cutoff of 156 Rydbergs and a convergence threshold of $1 \times 10^{-7}$ was applied to limit the error to within $5 \%$. The Brillouin zone was sampled by Monkhorst-Pack [7, $8]$ k-point meshes centered at the $\Gamma$ point. A mesh size of $4 \times 4 \times 4$ for the bulk structure calculations and a mesh size of $4 \times 4 \times 1$ for the surface calculations were used. A super-cell with 4 layers consisting of 120 atoms from the relaxed bulk structure with $10 \AA$ of vacuum space was created to study the surface properties.

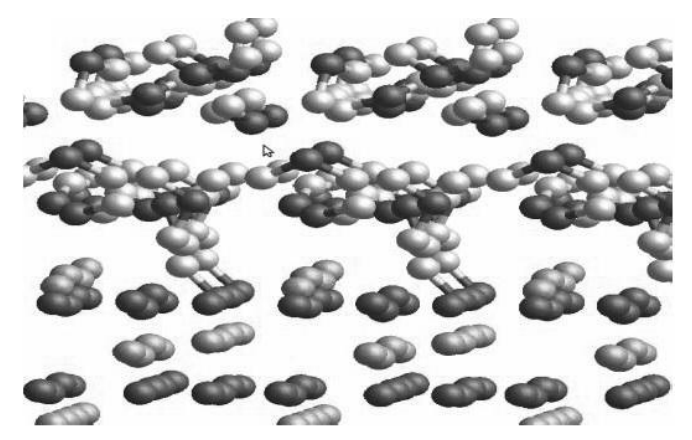

Fig. 2. Snapshot of the relaxed structure. Both molecular and dissociative adsorption processes are present. $\mathrm{Ga}$, $\mathrm{O}$, and $\mathrm{H}$ atoms are represented by grey, black and silver, respectively.

The hydration radius of a $\mathrm{Na}$ cation was calculated by finding the number of water molecules that relaxed within the first hydration sphere of the cation. The number of reactive surface sites on the oxide was obtained by minimizing the energy per absorbed water molecule. It was observed that the distance between the O-plane of the oxide and the first and second layers of adsorbed water was in close proximity of those of $\mathrm{Al}_{2} \mathrm{O}_{3}[3,9]$. Lattice parameters of the $\alpha-\mathrm{Ga}_{2} \mathrm{O}_{3}$ that 
were calculated in this study are summarized in Table 1 . The relaxed bond lengths and the average distance of the first and second layer of water to the oxide surface are summarized in Table 2. Fig. 2, depicts the energy minimum structure of the interface between the $\mathrm{Ga}_{2} \mathrm{O}_{3}$ surface and the first and second layer of water molecules.

Table I

lattice parameters

\begin{tabular}{|c|c|c|c|c|}
\hline Phase & Space group & This work & $\mathrm{DFT}^{\mathrm{a}}$ & Experiment $^{\mathrm{a}}$ \\
\hline$\alpha$ & $R \overline{3} c$ & $\begin{array}{l}a=4.981 \\
c=13.431\end{array}$ & $\begin{array}{l}a=5.059 \\
c=13.618\end{array}$ & $\begin{array}{l}a=4.983 \\
c=13.433\end{array}$ \\
\hline
\end{tabular}

Space groups and lattice parameters in $\AA$ of the $\alpha-\mathrm{Ga}_{2} \mathrm{O}_{3}$ structure. ${ }^{\text {a }}$ Reference[1]

Table II

Relaxed bond length

\begin{tabular}{c|c|c|l}
\hline Bond Length & This study & Other study & Experiment \\
\hline$R_{G a_{s}-O_{s}}$ & 1.84 & $1.845^{\mathrm{b}}$ & $1.921 \pm 0.010$ \\
\hline$R_{G a_{s}-O_{w}}$ & 1.848 & $2.099^{\mathrm{b}}$ & \\
\hline$R_{O_{w}-H_{w} \text { first layer }}$ & 1.022 & $0.986^{\mathrm{b}}$ & \\
\hline$R_{O_{w}-O_{w} \text { first layer }}$ & 1.559 & & \\
\hline$R_{O_{s}-O_{w} \text { first layer }}$ & 2.6539 & $2.3^{\mathrm{d}}$ & \\
\hline$R_{O_{s}-O_{w \text { second layer }}}$ & 5.4308 & $5.278^{\mathrm{d}}$ & \\
\hline $\begin{array}{c}\text { Hydration Radius } \\
\text { Na }\end{array}$ & 2.8 & $3.06^{\mathrm{c}}$ & \\
coordination number 6 & & & \\
\hline
\end{tabular}

Relaxed bond length in $\AA$. Values are obtained by averaging over 12 water atoms in the first and 12 water molecules in the second layer.

${ }^{b}$ Reference [2], ${ }^{\mathrm{c}}$ Reference[6] , ${ }^{\mathrm{d}} \mathrm{Al}_{2} \mathrm{O}_{3}$ parameters are mentioned for comparison $[8,9]$,

Sverjensky et al. [3] used the surface site density, $\mathrm{N}_{\mathrm{s}}$ obtained by isotopic exchange methods. In this study, it was observed that molecular adsorption of 4 water molecules on the gallium oxide surface minimized the energy of the system. Therefore, it is assumed that 4 sites are available on average per unit cell surface area of $4.31 \times 10^{-19} \mathrm{~m}^{2}$, which gives a surface site density of 9.28 site per $n m^{2}$. Fig. 3, depicts the changes of the surface adsorption energy against the number of adsorbed water molecules.

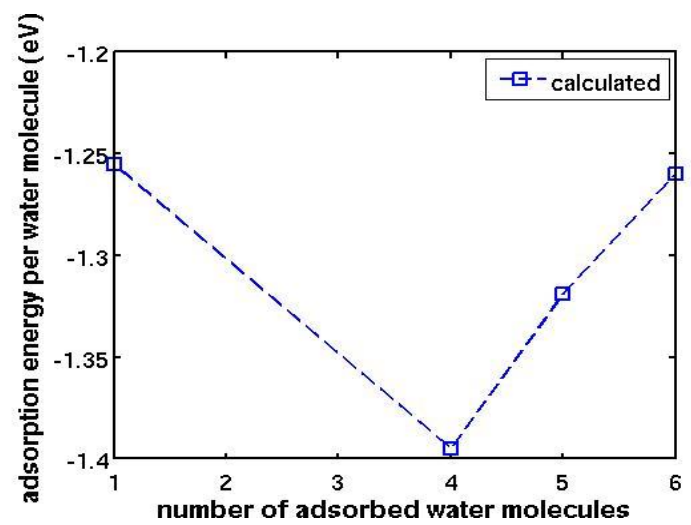

Fig. 3. Surface adsorption energy against the number of adsorbed water molecules

\section{B. Surface Dissociation Rates}

The oxide surface-electrolyte interface is approximated by an electrical double layer (DL) model. The surface complexation processes can be described as $[3,10]$

$$
\begin{gathered}
>\mathrm{SOH}+H_{a q}^{+} \Leftrightarrow>\mathrm{SOH}_{2}^{+} \\
K_{S, 1}=\frac{a_{>S O H_{2}^{+}}}{a_{S O H} a_{H_{a q}^{+}}} 10^{F \psi_{0} / 2.303 R T} \\
>S O^{-}+>H_{a q}^{+} \Leftrightarrow>S O H \\
K_{S, 2}=\frac{a_{>S O H}}{a_{>S O^{-}} a_{H_{a q}^{+}}} 10^{F \psi_{0} / 2.303 R T}
\end{gathered}
$$

where $>S$ represents the surface site which is bonded to the underlying bulk material, $a_{j}$ represents the activity of the $\mathrm{j}^{\text {th }}$ surface species, F represent Faraday's constant, R is the ideal gas constant, and $\mathrm{T}$ is the temperature. The power term in the equilibrium constant for equations 2 and 4 describes the electrostatic work required to bring the ion from the bulk to the relevant charged surface. The equilibrium process at the $\mathrm{pH}$ of the pristine point of zero charge $\left(\mathrm{pH}_{\mathrm{ppzc}}\right)$ is described by

$$
p H_{p p z c}=0.5\left(\log K_{s 2}+\log K_{s 1}\right)
$$

The difference between the surface protonation constants is defined as

$$
\Delta p K=\log K_{s 2}-\log K_{s 1}
$$

Born solvation theory of surfaces along with the electrostatics can be used to obtain the following relations to calculate $p H_{p p z c}$ and $\Delta p K[11]$

$$
\begin{gathered}
p H_{p p z c}=-0.5\left(\frac{\Delta \Omega_{r, Z}}{2.303 R T}\right)\left(\frac{1}{\epsilon_{k}}\right)-B_{Z}\left(\frac{s}{r_{>S-O H}}\right)+\log k_{H^{+}, Z}^{\prime \prime} \\
\Delta p K=\left(\frac{\Delta \Omega_{r, n}}{2.303 R T}\right)\left(\frac{1}{\epsilon_{k}}\right)-B_{n}\left(\frac{s}{r_{>S-O H}}\right)+\log k_{H^{+}, n}^{\prime \prime}
\end{gathered}
$$

where $\epsilon_{k}$ is the permittivity of the bulk oxide and $\frac{s}{r_{>S-O H}}$ is the Pauling bond strength per unit bond length for the oxide bulk cation, $\Delta \Omega_{r, z}$ is the interfacial Born solvation coefficient and represents the contribution from solvation to the free energy of surface protonation, $B_{z}$ is the electrostatic constant, and $k_{H^{+}, Z}^{\prime \prime}$ is related to intrinsic contribution. Sverjenski et al. fitted experimental points of zero charge from literature to the equations 7 and 8 to obtain

$$
\begin{gathered}
p H_{p p z c}=21.1158\left(\frac{1}{\epsilon_{k}}\right)-429148\left(\frac{s}{r_{>s-O H}}\right)+14.4866 \\
\Delta p K=12.692\left(\frac{s}{r_{>S-O H}}\right)+3.537
\end{gathered}
$$


Equations 5-10 are used to derive the point of zero charge and the surface dissociation constants. In calculating the Pauling bond strength per unit bond length, the valency of the Ga can be calculated using Shannon's equation [12]

$$
S=S_{0}\left(\frac{R}{R_{0}}\right)^{-N}
$$

\section{Surface Capacitance}

The distance of the first layer of water to the $O_{s}-$ surface i.e. $R_{O_{s}-O_{w f i r s t l a y e r}}$ and the cation hydration radii that were calculated through DFT calculations are used to calculate the interfacial capacitance $\left(\mu F . \mathrm{cm}^{-2}\right)$ between the surface and the first layer of water

$$
\frac{1}{C}=\frac{R_{\text {hydrated cation }}}{8.854 \varepsilon_{\text {int }}}+\frac{R_{O_{S}-} O_{w \text { first layer }}}{8.854 \varepsilon_{\text {int }}}
$$

Table 3, summarizes the calculated parameters and lists experimental and simulated values for comparison, where available. The atomic coordinates from the relaxed surfacewater interface are used to calculate four out of the five parameters that are required to calculate surface speciation which are the surface dissociation constants, number of surface sites and the capacitance of the first layer. The second layer is considered as a constant capacitance [3]. The values of the calculated point of zero charge, and surface dissociation constants were in close agreement with the experimental values .

Table III

\begin{tabular}{|c|c|c|c|}
\hline & $\mathrm{Ga}_{2} \mathrm{O}_{3}$ & other study & $\mathrm{Al}_{2} \mathrm{O}_{3}^{\dagger}$ \\
\hline$r_{>S-O} \quad[\AA]$ & 2.6539 & & 1.913 \\
\hline$r_{>S-O H}[\AA]$ & $2.6539+1.01$ & & $1.913+1.01$ \\
\hline$S$ & 0.75 & & 0.5 \\
\hline$S / r_{>S-O H}$ & 0.2047 & & 0.1710 \\
\hline$\varepsilon_{k}$ & 10.2 & & 10.43 \\
\hline$\varepsilon_{\text {int }}$ & $53^{*}$ & & 53 \\
\hline$C\left[\mu F . \mathrm{cm}^{-2}\right]$ & 86.0415 & & 99 \\
\hline$p H_{p p z c}$ & 7.7721 & $8^{+}, 9^{ \pm}$ & 8.6 \\
\hline$\Delta p K$ & 6.1351 & & 6 \\
\hline $\log K_{2}$ & 4.7046 & $6^{+}$ & 5.5 \\
\hline $\log K_{1}$ & 10.8396 & $10^{+}$ & 11.5 \\
\hline$N_{s}\left[\right.$ sites. $\left.\mathrm{nm}^{-2}\right]$ & 9.28 & $9^{+}$ & 8 \\
\hline
\end{tabular}

Calculated parameters of the surface adsorption

${ }^{\dagger}$ Reference $[2,3], \mathrm{Al}_{2} \mathrm{O}_{3}$ parameters are mentioned for comparison, *Interfacial permittivity of $\mathrm{Ga}_{2} \mathrm{O}_{3}$ is assumed to be equal to that of $\mathrm{Al}_{2} \mathrm{O}_{3}$. Capacitance is calculated for $\mathrm{NaCl}$ solution,

${ }^{ \pm}$Reference [4], Experiment, ${ }^{+}$Reference[8], Simulated,

\section{Surface Charging Mechanism}

Despite the heterogeneous nature of the oxide surfaces, for simplification purposes, it can be assumed that all surface sites are equivalent [3]. Any surface atom capable of adsorbing or desorbing a proton is considered a surface site [2]. Therefore, mass balance on surface sites requires

$$
N_{T}=\left(c_{>S_{O H}^{+}}+c_{>S O H}+c_{>S^{-}}\right)
$$

where $N_{T}$ represents the total number of surface sites. Surface charge $\left(\mathrm{C} . \mathrm{m}^{-2}\right)$ at $\mathrm{O}$-plane is calculated through

$$
\sigma_{0}=\frac{F}{A C_{s}}\left(c_{>\mathrm{SOH}_{2}^{+}}-c_{>\mathrm{SO}^{-}}\right)
$$

It can be assumed that the oxide-electrolyte interface behaves like a parallel plate capacitor where the charge and potential are related through

$$
\sigma_{0}=C\left(\psi_{0}-\psi_{d}\right)
$$

where $\psi_{0}$ represents the surface potential and $\psi_{d}$ represents the Stern potential. For a 1:1 $\left(\mathrm{M}^{+} \mathrm{L}^{-}\right)$electrolyte, electrical charge at the hypothetical parallel planes can be obtained using the modified Grahame equation [10]

$$
\sigma_{0}=\left(8 \varepsilon_{0} \varepsilon_{w} K_{B} T C^{0}\right)^{1 / 2} \sinh \left(\frac{q z \psi_{d}}{2 K_{B} T}\right)
$$

where $\varepsilon_{0}$ and $\varepsilon_{w}$ represent the permittivity of the free space and the aqueous medium respectively, $K_{B}$ is the Boltzmann's constant, $Z$ is the ion valence number and $C^{0}$ is the bulk concentration of the solution (particles. $\mathrm{m}^{-3}$ ). The surface charge density can be connected to the surface dissociation reactions by [13]

$$
\sigma_{0}=q N_{S}\left(\frac{10^{-2 p H} \exp \left(\frac{-q \psi_{0}}{K_{B} T}\right)-K_{S 1} K_{S 2} \exp \left(\frac{q \psi_{0}}{K_{B} T}\right)}{K_{S 1} 10^{-p H}+10^{-2 p H} \exp \left(\frac{-q \psi_{0}}{K_{B} T}\right)+K_{S 1} K_{S 2} \exp \left(\frac{q \psi_{0}}{K_{B} T}\right)}\right)
$$

Equations 15-17 can be solved numerically to obtain the surface potential. Fig. 4, depicts the experimental and calculated surface potential on the O-plane using equations $1-17$. It can be observed that the simulated values are in close agreement with the experiment

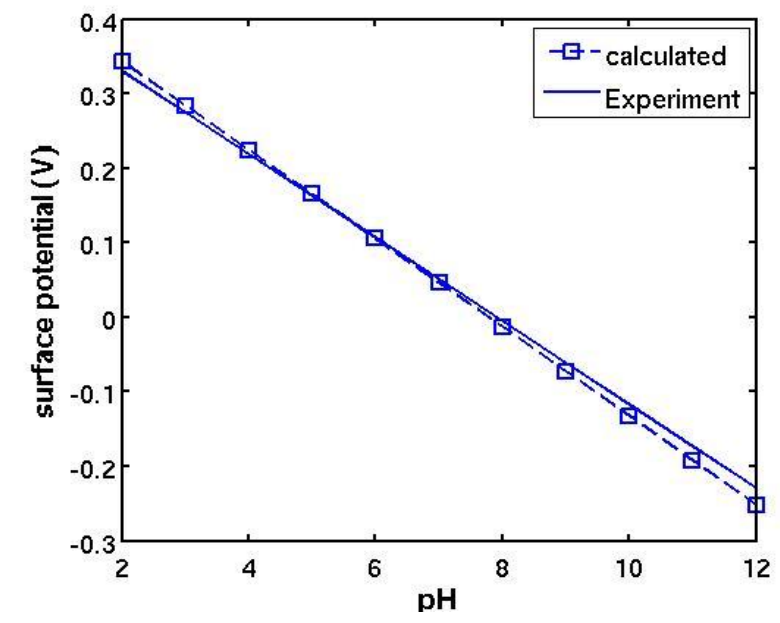

Fig. 4. Calculated surface charge as a function of $\mathrm{pH}$ 


\section{E. Sensitivity}

An analytical model [14] is used to simulate the sensitivity of the device to the changes of $\mathrm{pH}$. The device characteristics are the same as those of the device studied in reference [10, 15]. The $\mathrm{AlGaN} / \mathrm{GaN}$ heterostructure is grown on sapphire by metal-organic vapor phase epitaxy with aluminum composition of 0.23 and $\mathrm{AlGaN}$ thickness of $22 \mathrm{~nm}$. The gate length is $1 \mu \mathrm{m}$ and the gate width is $6 \mu \mathrm{m}$. The electron mobility and density of the two dimensional electron gas are $950 \mathrm{~cm}^{2} / \mathrm{V}$ and $8.0 \times 10^{12} \mathrm{~cm}^{-2}$, respectively. The doping concentration is $0.5 \times 10^{18} \mathrm{~cm}^{-3}$. The contact resistance and the saturation velocity are assumed to be $10 \Omega$ and $1 \times 10^{7}$ $\mathrm{cm} . \mathrm{s}^{-1}$ respectively. Fig. 5, depicts the calculated and experimental values. An offset value of $8 \mathrm{~mA}$ is present between the simulated and experimental data. The experimental and simulated results show a sensitivity of 37 $\mu \mathrm{A}$ per $\mathrm{pH}$ unit and $41 \mu \mathrm{A}$ per $\mathrm{pH}$ unit respectively when $\mathrm{V}_{\mathrm{ds}}$ $=0.2 \mathrm{~V}$ and $\mathrm{V}_{\mathrm{gs}}=-0.5 \mathrm{~V}$.

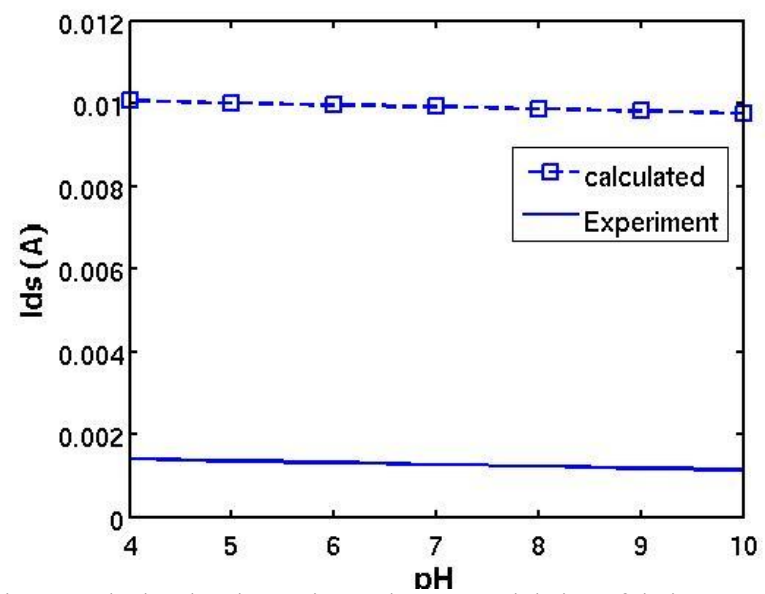

Fig. 5. Calculated and experimental[7,11] modulation of drain current as a function of $\mathrm{pH}$ at $\mathrm{Vds}=0.2 \mathrm{~V}$

\section{CONCLUSION}

A complete description of the surface charging mechanism has been given. The relaxed bond-lengths that were obtained through $a b$ initio calculations were used to calculate the surface reaction rates, interfacial capacitance and surface site density and all these characteristics were in close agreement with experimental values. A double layer model was used to calculate the surface charge and potential. The calculated surface potential was in close agreement with the experiment. An analytical approach was used to calculate the device current - voltage sensitivity to the changes in $\mathrm{pH}$. There was an offset value observed between the simulated results and the experiment that needs to be further investigated. However, the calculated sensitivity of the sensor was in close agreement with the experiment.

\section{ACKNOWLEDGMENT}

We acknowledge the support from the Australian Research Council (Discovery Projects).This work was also supported by IVEC through the use of advanced computing resources located atiVEC@Murdoch.

\section{REFERENCES}

[1] S. Yoshioka, H. Hayashi, A. Kuwabara, F. Oba, K. Matsunaga, and I. Tanaka, "Structures and energetics of Ga2O3 polymorphs," Journal of Physics: Condensed Matter, vol. 19, p. 346211, 2007.

[2] X. Zhou, E. J. Hensen, R. A. van Santen, and C. Li, "DFT Simulations of Water Adsorption and Activation on Low-Index a-Ga2O3 Surfaces," Chemistry-A European Journal, vol. 20, pp. 6915-6926, 2014.

[3] D. A. Sverjensky, "Prediction of surface charge on oxides in salt solutions: Revisions for 1: $1\left(\mathrm{M}^{+} \mathrm{L}^{-}\right)$electrolytes," Geochimica et Cosmochimica Acta, vol. 69, pp. 225-257, 2005.

[4] M. Kosmulski, "Pristine points of zero charge of gallium and indium oxides," Journal of colloid and interface science, vol. 238, pp. 225-227, 2001.

[5] J. P. Perdew and A. Zunger, "Self-interaction correction to density-functional approximations for many-electron systems," Physical Review B, vol. 23, p. 5048, 1981.

[6] S. B. Rempe and L. R. Pratt, "The hydration number of $\mathrm{Na}^{+}$in liquid water," Fluid Phase Equilibria, vol. 183, pp. 121-132, 2001.

[7] H. J. Monkhorst and J. D. Pack, "Special points for Brillouin-zone integrations," Physical Review B, vol. 13, p. 5188, 1976.

[8] M. Bayer, C. Uhl, and P. Vogl, "Theoretical study of electrolyte gate AlGaN GaN field effect transistors," Journal of applied physics, vol. 97, p. 033703, 2005.

[9] M.-P. Gaigeot, M. Sprik, and M. Sulpizi, "Oxide/water interfaces: how the surface chemistry modifies interfacial water properties," Journal of Physics: Condensed Matter, vol. 24, p. 124106, 2012.

[10] S. Rabbaa and J. Stiens, "Validation of a triangular quantum well model for GaN-based HEMTs used in $\mathrm{pH}$ and dipole moment sensing," Journal of Physics D: Applied Physics, vol. 45, p. 475101, 2012.

[11] D. A. Sverjensky and N. Sahai, "Theoretical prediction of singlesite enthalpies of surface protonation for oxides and silicates in water," Geochimica et cosmochimica acta, vol. 62, pp. 37033716, 1998.

[12] I. t. Brown and R. Shannon, "Empirical bond-strength-bondlength curves for oxides," Acta Crystallographica Section A: Crystal Physics, Diffraction, Theoretical and General Crystallography, vol. 29, pp. 266-282, 1973.

[13] R. Van Hal, J. Eijkel, and P. Bergveld, "A general model to describe the electrostatic potential at electrolyte oxide interfaces," Advances in Colloid and Interface Science, vol. 69, pp. 31-62, 1996.

[14] H. Morkoç, Handbook of Nitride Semiconductors and Devices, Materials Properties, Physics and Growth vol. 1: John Wiley \& Sons, 2009.

[15] T. Kokawa, T. Sato, H. Hasegawa, and T. Hashizume, "Liquidphase sensors using open-gate $\mathrm{AlGaN} / \mathrm{GaN}$ high electron mobility transistor structure," Journal of Vacuum Science \& Technology B: Microelectronics and Nanometer Structures, vol. 24, pp. 19721976, 2006. 